\title{
Multiple flux jumps and irreversible behavior of thin Al superconducting rings
}

\author{
D. Y. Vodolazov and F. M. Peeters* \\ Departement Natuurkunde, Universiteit Antwerpen (UIA), Universiteitsplein 1, B-2610 Antwerpen, Belgium \\ S. V. Dubonos ${ }^{\dagger}$ and A. K. Geim \\ Department of Physics and Astronomy, University of Manchester, Oxford Road, M13 9PL, Manchester, United Kingdom
}

(Received 11 September 2002; published 21 February 2003)

\begin{abstract}
An experimental and theoretical investigation was made of flux jumps and irreversible magnetization curves of mesoscopic Al superconducting rings. In the small magnetic-field region the change of vorticity with magnetic field can be larger than unity. This behavior is connected with the existence of several metastable states of different vorticities. The intentional introduction of a defect in the ring has a large effect on the size of the flux jumps. Calculations based on the time-dependent Ginzburg-Landau model allows us to explain the experimental results semiquantitatively.
\end{abstract}

DOI: 10.1103/PhysRevB.67.054506

PACS number(s): 74.25.Op, 74.20.De, 73.23.-b

\section{INTRODUCTION}

Recently, Pedersen et al. ${ }^{1}$ observed jumps in the magnetization of superconducting rings which corresponds to changes of the vorticity larger than unity. This is in contrast to the behavior of superconducting disks where only changes of the vorticity with unit size were observed. ${ }^{2}$ In some respect the observed behavior in rings is similar to vortex avalanches which were observed in superconductors with strong bulk pinning ${ }^{3,4}$ or to jumps in the magnetization when several vortices (in the form of a chain) enter into a superconducting film of width comparable to the coherence length., The occurrence of such jumps in a defect-free superconducting ring originates from the fact that several metastable states with different vorticities $L$ are stable at a given magnetic field. However the existence of such multiple stable states is not a sufficient condition to explain changes in the vorticity larger than unity (e.g., they also exist in the case of superconducting disks). An additional important requirement is to find the stability condition for those metastable states and to determine the state to which the system relaxes. This requires the study of the transition process from one state to another, i.e., it requires analyzing time-dependent processes.

The stability condition was studied numerically in Ref. 7 for the case of a hollow cylinder, and in a number of works (see, for example, Refs. 8 and 9, and references therein) for superconducting disks and rings by using the static Ginzburg-Landau (GL) equations. Unfortunately no analytical results were presented due to the rather general character of the studied systems in the above works.

Recently, we studied the transition process ${ }^{10}$ between states with different vorticities using the time-dependent Ginzburg-Landau equations. It was shown that transitions between different metastable states in a mesoscopic superconducting ring are governed by the ratio between the time relaxation of the phase of the order parameter $\tau_{\phi}$ (which is inversely proportional to the Josephson frequency) and the time relaxation of the absolute value of the order parameter $\tau_{|\psi|}$. We found that if the ratio $\tau_{|\psi|} / \tau_{\phi}$ is sufficiently large the system will always transit from a metastable state to the ground state. This leads to an avalanche-type variation of $L$ when the vorticity of the metastable state differs appreciably from the vorticity of the ground state. In contrast to the case of a superconducting film, in a ring the "vortex" entry occurs through a single point and the vorticity increases one by one during the transition. In low-temperature superconductors such as $\mathrm{In}, \mathrm{Al}$, and $\mathrm{Sn}$ the ratio $\tau_{|\psi|} / \tau_{\phi}$ is very large for temperatures far below the critical temperature $T_{c}$ and hence, if such systems are driven far out of equilibrium they will always relax to the ground state.

In this work we investigate the conditions under which a state with a given vorticity becomes unstable in a finite width ring and we find how the superconducting order parameter in the ring changes with increasing applied magnetic field. We are able to find an analytical expression for the dependence of the order parameter on applied magnetic field, and hence for the upper critical field at which superconductivity vanishes in such a sample. We provide a direct comparison of the theoretical and experimental results on aluminum rings. Our theoretical calculations are based on a numerical solution of the time-dependent Ginzburg-Landau equations.

The paper is organized as follows. In Sec. II the theoretical formalism is presented and the two-dimensional timedependent GL equations are solved. In Sec. III the experimental results are presented and compared with our theory. In Sec. IV we present our conclusions and our main results.

\section{THEORY}

We consider sufficiently narrow rings such that we can neglect screening effects. This is allowed when the width of the ring $w$ is less than $\max \left(\lambda, \lambda^{2} / d\right)$, where $\lambda$ is the London penetration length and $d$ is the thickness of the ring. In order to study the response of such a ring on the applied magnetic field we use the time-dependent Ginzburg-Landau equations

$$
\begin{gathered}
u\left(\frac{\partial \psi}{\partial t}+i \varphi \psi\right)=(\nabla-i \mathbf{A})^{2} \psi+\left(1-|\psi|^{2}\right) \psi, \\
\Delta \varphi=\operatorname{div}\left\{\operatorname{Im}\left[\psi^{*}(\nabla-i \mathbf{A}) \psi\right]\right\},
\end{gathered}
$$


where all the physical quantities (order parameter $\psi$ $=|\psi| e^{i \phi}$, vector potential $A$, and electrostatical potential $\varphi$ ) are measured in dimensionless units: the vector potential $A$ is scaled in units $\Phi_{0} /(2 \pi \xi)$ (where $\Phi_{0}$ is the quantum of magnetic flux), and the coordinates are in units of the coherence length $\xi(T)$. In these units the magnetic field is scaled by $H_{c 2}$ and the current density, $j$, by $j_{0}=c \Phi_{0} / 8 \pi^{2} \lambda^{2} \xi$. Time is scaled in units of the Ginzburg-Landau relaxation time $\tau_{G L}$ $=4 \pi \sigma_{n} \lambda^{2} / c^{2}$, and the electrostatic potential, $\varphi$, is in units of $c \Phi_{0} / 8 \pi^{2} \xi \lambda \sigma_{n}\left(\sigma_{n}\right.$ is the normal-state conductivity). Here the time derivative is explicitly included which allows us to determine the moment at which the state with given vorticity $L$ becomes unstable. The coefficient $u$ governs the relaxation of the order parameter. For example, the time relaxation of the absolute value of the order parameter varies as $\tau_{|\psi|}$ $\sim u^{0.6}$ and the time relaxation of the phase of the order parameter (which is inversely proportional to the time of the charge imbalance $\tau_{Q} \sim 1 / \sqrt{\tau_{\phi}}$ ) as $\tau_{\phi} \sim u^{0.22}$ (see Ref. 10).

It is essential to include the electrostatic potential (which is responsible for the appearance of the Josephson time or frequency) in order to account for multivortex jumps. First, it is connected with the conversion of superconducting current into normal current and vice versa, which implies for the full current density in the superconducting ring that $\operatorname{div}\left(j_{s}+j_{n}\right)$ $=0$, which reflects conservation of the total current in space [with $j_{s}=\operatorname{Im}\left\{\psi^{*}(\nabla-i \mathbf{A}) \psi\right\}$ the superconducting and $j_{n}$ $=-\nabla \varphi$ the normal current density]. Secondly, it leads to different time relaxation scales for the phase and magnitude of the order parameter. As a result multivortex jumps in the ring become possible if the ratio ${ }^{10} \tau_{|\psi|} / \tau_{\phi} \gg 1$. In some previous studies (see, for example, Refs. 11 and 12) $\varphi=0$ was assumed and as a consequence only transitions with unit vorticity jumps, i.e., $\Delta L=1$, are possible in the ring ${ }^{10}$ because in this case $\tau_{|\psi|} / \tau_{\phi} \sim 1$ at any value of the parameter $u$ and radii of the ring. In our calculations we choose the value $u$ $=48$ which ensures the condition $\tau_{|\psi|} / \tau_{\phi} \gg 1$.

We assume that the width $(w)$ of the ring is less than two coherence lengths $\xi$, because (i) all experimental results presented here were performed for such samples and (ii) only in this case it is possible to obtain simple analytical expressions. For instance, this is the case for the dependence of the order parameter on the applied magnetic field and the upper critical field $H_{\max }$.

For $w \leqslant 2 \xi$ the order parameter is practically independent of the radial coordinate. This is demonstrated in Fig. 1 where the dependence of the order parameter in the middle of the ring is compared with its value at the inner and outer boundaries of the ring, i.e., $r=R \pm w / 2$ ( $R$ are the mean radii of the rings), for two different rings. Notice that these two numerical examples correspond already to relatively wide mesoscopic rings, i.e., $R / w \sim 1-2$. For the field $H_{\max }$ we are able to fit our numerical results to the expression

$$
H_{\max }=3.67 \frac{\Phi_{0}}{2 \pi \xi w} .
$$

For rings with $w \leqslant 2 \xi$ and $w / R<1$ this analytical expression is within $2 \%$ of the numerical results. It is interesting to note that $H_{\max }$ does not depend on the radii of the ring. But the

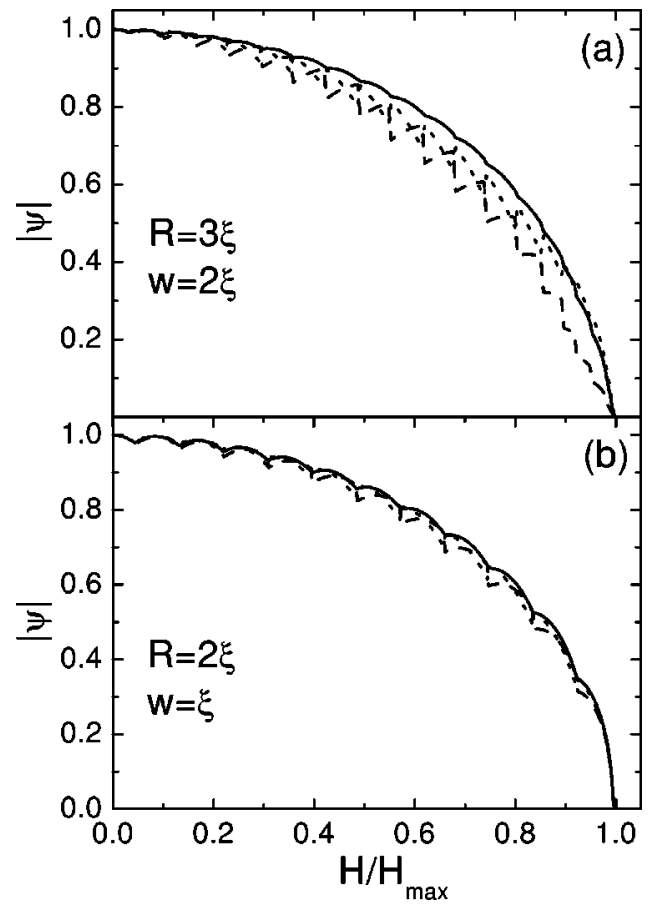

FIG. 1. Dependence of the absolute value of the order parameter $|\psi|$ on the applied magnetic field for two different sizes of the rings in the ground state. Dashed curves correspond to $|\psi|(R-w / 2, H)$, solid curves to $|\psi|(R, H)$, and the dotted curve to $|\psi|(R$ $+w / 2, H)$.

value of the vorticity of the system depends on $R$. For example, for $R=5.5 \xi(16.5 \xi)$ we have $L=55(501)$ for $w=\xi$ at $H=H_{\max }$.

Note that Eq. (2) has the same dependence on the superconducting parameters as the case of a thin plate with thickness $d<\sqrt{5} \lambda$ placed in a parallel magnetic field. ${ }^{13,14}$ Even the numerical coefficient is quite close, i.e., for a thin plate it is equal to $2 \sqrt{3} \simeq 3.46$. Furthermore, we found that the transition to the normal state of our rings at the critical field $H_{\text {max }}$ is of second order as is also the case for a thin plate. A possible reason for this close similarity is that for a thin plate with thickness $d<\sqrt{5} \lambda$ the screening effects are also very small. In the calculations of Refs. 13 and 14 an average value for the order parameter was used independent of the coordinate. Note that this is similar to our $|\psi|$ which is practically independent of the radial coordinate (see Fig. 1).

The absolute value of the order parameter (in the middle of the ring) is, to a high accuracy, given by the expression

$$
|\psi|^{2}=1-\left(H / H_{\max }\right)^{2}-p(L, H)^{2},
$$

with $p(L, H)=L / R-H R / 2$, where the vorticity $L$ depends on the history of the system. This result is similar to the one obtained in Refs. 13 and 14 with the exception of the last term in Eq. (3) which appears due to the closed geometry of the ring and hence leads to a nonzero $L$.

All the above results were obtained for a ring which is in the ground state at any value of the magnetic field. However, such a system can exhibit several metastable states at a given magnetic field, and consequently this may lead to hysteretic behavior when one sweeps the magnetic field up and down. 
Furthermore, with changing field the vorticity may jump with $\Delta L>1$. An important question which arises is the condition of stability of the state with given vorticity. This question was studied earlier for one-dimensional rings, ${ }^{12,15}$ i.e., rings with zero width. It turns out that the system transits to a state with another vorticity when the value of the gaugeinvariant momentum $\mathbf{p}=\nabla \phi-\mathbf{A}$ reaches the critical value

$$
p_{c}=\frac{1}{\sqrt{3}} \sqrt{1+\frac{1}{2 R^{2}}} \text {. }
$$

Physically, it means that at this value the kinematical energy of the Cooper pairs becomes similar to their binding energy. Using Eq. (4) it is easy to find the value of the magnetic field for the first "vortex" entry,

$$
H_{e n} / H_{c 2}=2 p_{c} / R=\frac{2}{\sqrt{3} R} \sqrt{1+\frac{1}{2 R^{2}}} .
$$

We will now generalize the results of Refs. 12 and 15 to the case of rings with finite width but such that $w \leq 2 \xi$. First we will neglect the dependence of $\psi$ on the radial coordinate in which case the GL equations reduce to one-dimensional expressions. But in order to include the suppression of the order parameter by an external field for a finite width ring we add the term $-\left(H / H_{\max }\right)^{2} \psi$ to the right-hand side of Eq. (1a), where $H_{\max }$ is given by Eq. (2). Using the stability analysis of the linearized Ginzburg-Landau equations near a specific metastable state as presented in Ref. 15 we obtain the modified critical momentum

$$
p_{c}=\frac{1}{\sqrt{3}} \sqrt{1-\left(\frac{H}{H_{\max }}\right)^{2}+\frac{1}{2 R^{2}}} .
$$

Note that now $p_{c}$ decreases with increasing magnetic field. This automatically leads to a decreasing value of the jump in the vorticity $\Delta L$ at high magnetic field, because in Ref. 10 it was shown that

$$
\Delta L_{\max }=\operatorname{Nint}\left(p_{c} R\right),
$$

where $\operatorname{Nint}(x)$ returns the nearest integer to the argument.

In order to check the validity of Eq. (6) we performed a numerical simulation of the two-dimensional GinzburgLandau equations, Eqs. (1a) and (1b), for a ring with $R$ $=5.5 \xi$ and $w=1.5 \xi$ (these parameters corresponds to the experimental situation-see the following section). In Fig. 2 the magnetization, the order parameter, and the gaugeinvariant momentum $p$ are shown as functions of the applied magnetic field. The magnetic field was cycled up and down from $H<-H_{\max }$ to $H>H_{\max }$. The condition (6) leads to a hysteresis of $M(H)$ and to a changing value of the jump in the vorticity in accordance with the change in $p_{c}$. The main difference between our theoretical prediction (6) and the results of our numerical calculations appears at fields close to $H_{\max }$. Apparently it is connected with the fact that for the considered ring the distribution of the order parameter along

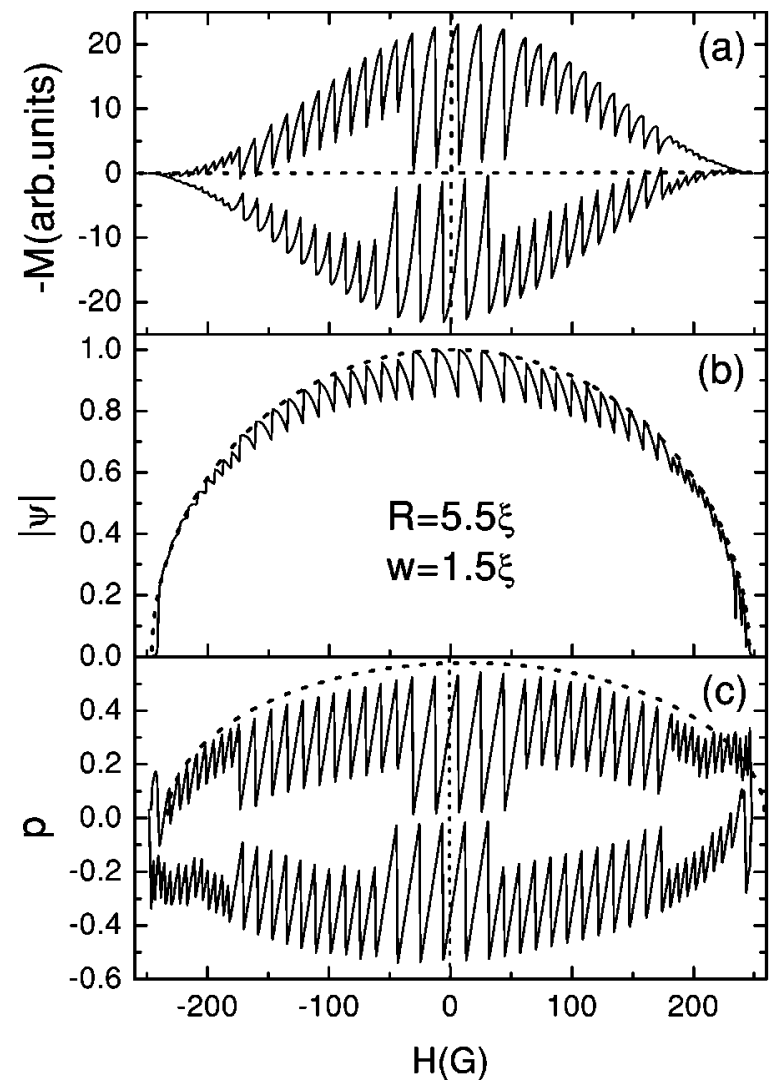

FIG. 2. Magnetic-field dependence of the magnetization (a), the order parameter (b), and the gauge-invariant momentum (c) in the middle of the ring. Dotted curve in (b) is the expression $\sqrt{1-\left(H / H_{\max }\right)^{2}}$. Dotted curve in (c) is the expression $\sqrt{1-\left[\left(H-H_{0}\right) / H_{\max }\right]^{2}} / \sqrt{3}$, where $H_{0} \simeq 13 G$ is the displacement of the maximum of $M(H)$ from the $H=0$ line.

the width of the ring is appreciably nonuniform at $H$ $\simeq H_{\max }$ and as a consequence the one-dimensional model breaks down (see Fig. 1).

Finally, we also considered the same ring with a defect. The effect of the defect was modeled by introducing in the right-hand side of Eq. (1a) the term $-\rho(s) \psi(s$ is the arc coordinate) where $\rho(s)=-1$ inside the defect region with size $\xi$ and $\rho(s)=0$ outside. This leads to the results shown in Fig. 3 for $M(H),|\psi|(H)$, and $p(H)$. Due to the presence of the defect, $p_{c}$ differs from Eq. (6) already at low magnetic field $\left[p_{c}(H=0) \simeq 0.33\right.$ at a given "strength" of the defect) and as a result only jumps with $\Delta L=1$ are possible in such a ring. In this case the $p_{c}$ and $|\psi|$ also depend on the applied magnetic field with practically the same functional dependence on $H$ as Eq. (6).

\section{COMPARISON WITH EXPERIMENT}

The measurements were performed on individual $\mathrm{Al}$ superconducting rings by using ballistic Hall micromagnetometry. ${ }^{16,17}$ The technique employs small Hall probes microfabricated from a high-mobility twodimensional electron gas. The rings-having radii $R$ $\simeq 1 \mu \mathrm{m}$ and width $w$ ranging from 0.1 to $0.3 \mu \mathrm{m}-$ were 


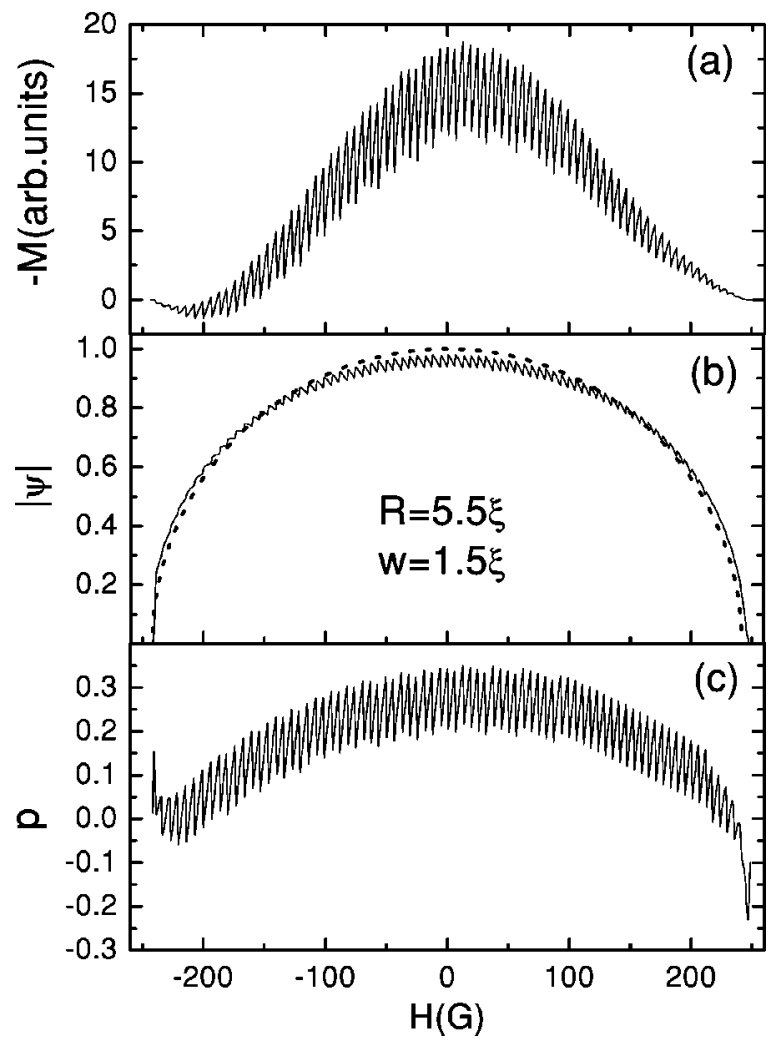

FIG. 3. Magnetic-field dependence of the magnetization (a), the order parameter (b), and the gauge-invariant momentum (c) (in the middle of the ring) of a ring containing a single defect. Dotted curve in (b) is the expression $\sqrt{1-\left(H / H_{\max }\right)^{2}}$.

placed directly on top of the microfabricated Hall crosses, which had approximately the same width $b$ of about $2 \mu \mathrm{m}$ (see micrograph in Fig. 4 for a ring with an artificial defect). These experimental structures were prepared by multistage

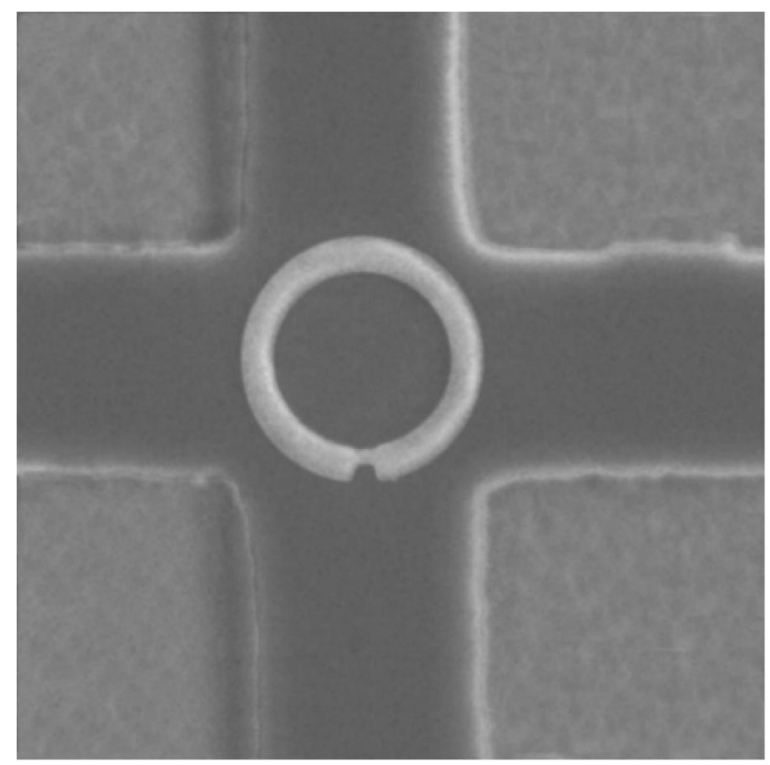

FIG. 4. A micrograph of the superconducting ring placed on top of a Hall bar. An artificial defect (narrowing of the ring cross section) is intentionally made by electron-beam lithography.

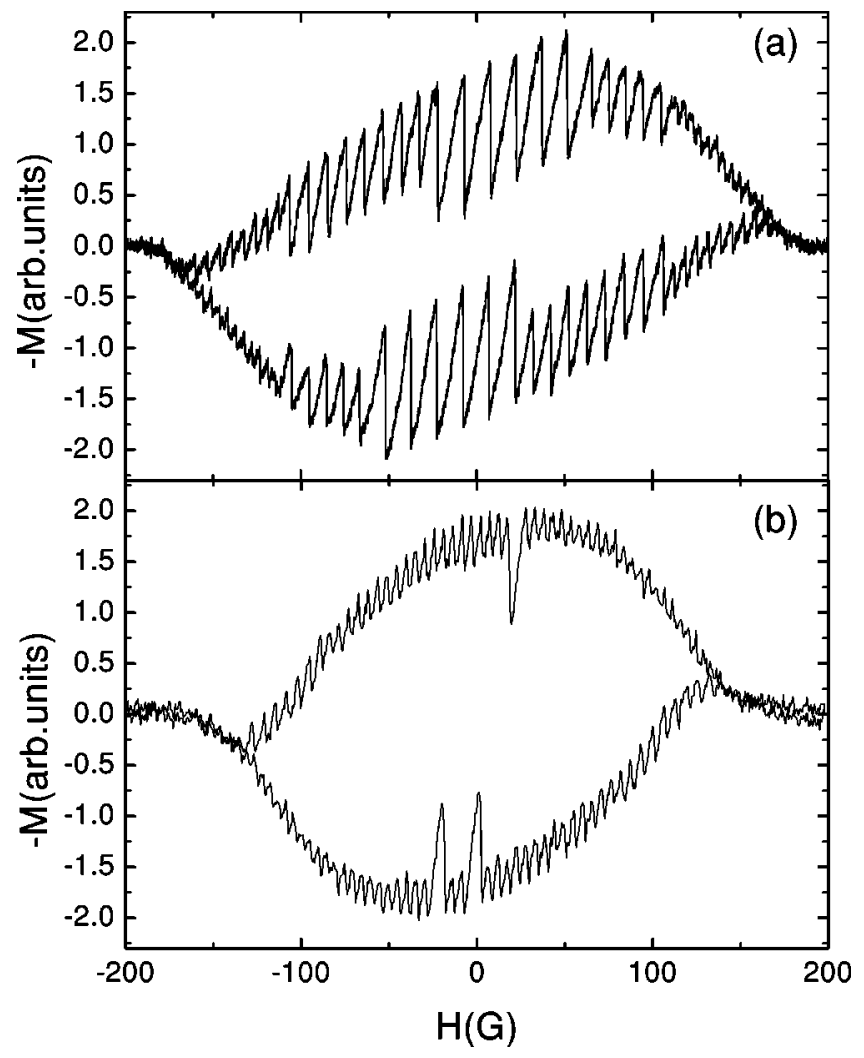

FIG. 5. Magnetic-field dependence of the magnetization of the ring without (a) and with (b) an artificial defect at $T \simeq 0.4 \mathrm{~K}$. Parameters of the rings (width and radii) are the same to within experimental accuracy.

electron-beam lithography with the accuracy of alignment between the stages better than $100 \mathrm{~nm}$. The rings studied in this work were thermally evaporated and exhibited a superconducting transition at about $1.25 \mathrm{~K}$. The superconducting coherence length was $\xi(T=0) \simeq 0.18 \mu \mathrm{m}$. The latter was calculated from the electron mean free path $l \simeq 25 \mathrm{~nm}$ of macroscopic Al films evaporated simultaneously with the $\mathrm{Al}$ rings. The Hall response, $R_{x y}$, of a ballistic cross is given by the amount of magnetic flux $\int B d s$ through the central square area $b \times b$ of the cross. ${ }^{16,18}$ For simplicity, one can view the ballistic magnetometer as an analog of a microsuperconducting quantum interference device, which would have a square pickup loop of size $b$ and superconducting rings placed in its center. We present our experimental data in terms of the area magnetization $M=\langle B\rangle-H$ which is the difference between the applied field $H$ and the measured field $\langle B\rangle \sim R_{x y}$. Previously, we have studied individual superconducting and ferromagnetic disks and found excellent agreement with the above formula. ${ }^{17,19}$ For further details about the technique, we refer the reader to our earlier work. ${ }^{16-18}$

Rings with and without an artificial defect were studied. Let us consider first the ring without an artificial defect. In Fig. 5(a) the full magnetization loop of such a ring with parameters $R=1.0 \pm 0.1 \mu \mathrm{m}$ and $w=0.25 \pm 0.05 \mu \mathrm{m}$ is shown. In Fig. 6 (solid curve) the low-field part of the virgin curve is presented. From the virgin trace $M(H)$ we can find the magnetic field for the first vortex entry, $H_{e n}$, and hence 


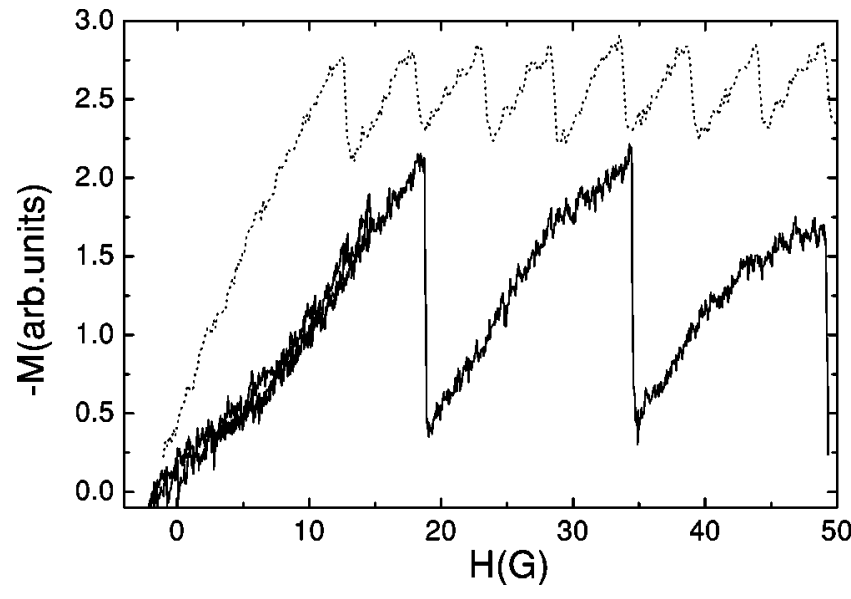

FIG. 6. Magnetic-field dependence of the virgin magnetization of a ring without (solid curve) and with (dotted curve) an artificial defect. The dotted curve is shifted for clarity by 0.6 .

we estimate $\xi \simeq 0.19 \mu \mathrm{m}$ at the given temperature ( $T$ $\simeq 0.4 \mathrm{~K}$ ) using Eq. (5) (this value of $\xi$ is in agreement with the above experimental value $\xi(0) \simeq 0.18 \mu \mathrm{m}$ obtained from the mean free path). Furthermore, we know from Fig. 5(a) that the vorticity changes with $\Delta L=3$ for $H \simeq 0$. This agrees with the fact that the radii of the ring are larger than $4.6 \xi$ [see Eq. (7)]. Another important piece of information which may be extracted from the virgin curve is that at the first vortex entry the magnetization drops considerably but it does not change sign. If we recall that at every vortex entry $p$ decreases on $1 / R$ (and hence the current density $j$ and $M$ $\sim \int[\mathbf{j} \times \mathbf{r}] d V$ also changes proportionally) we can conclude that the radii of our ring should be in the range $5.5 \xi \leq R$ $\leq 6.5 \xi$. This agrees with the experimental value $R / \xi \simeq 5.3$ \pm 0.5 .

If we take the above value for $\xi$ and $w \simeq 1.5 \xi$ we obtain the maximum field of $H_{\max } \simeq 223 \mathrm{G}$. This value is slightly smaller than the value obtained from Figs. 2 and $3, H_{\max }$ $\simeq 240 \mathrm{G}$, which we attribute to the large coordinate step that we used in our numerical calculations of Eqs. (1a) and (1b). The value is also larger than the experimental value $H_{\max }$ $\simeq 185 \mathrm{G}$. This disagreement between theory and experiment is most likely connected to the semiquantitative applicability of the Ginzburg-Landau equations in the considered temperature range. The range of applicability of the Ginzburg-Landau equations (even the stationary ones) for this specific superconductor is very narrow. Nevertheless based on previous comparison between experiments and theory for mesoscopic superconducting disks ${ }^{20,21}$ it was found that the GL equations provided a rather good description of the superconducting state even deep inside the $(H, T)$ phase diagram.

Figures 2(a) and 5(a) are qualitatively very similar. For example, our theory describes (i) the hysteresis; (ii) the nonunity of the vorticity jumps, i.e., $\Delta L=3$ in the low magnetic-field region, $\Delta L=2$ in the intermediate $H$ region, and $\Delta L=1$ near $H_{\max }$. Theoretically (experimentally) we found 6(5), 13(21), and 22(18) jumps with, respectively, $\Delta L=3,2$, and 1. (iii) Last, we found the nonsymmetric magnetization near $\pm H_{\max }$ for magnetic-field sweeps up and down.

In the ring with approximately the same mean radii and width but containing an intentionally introduced artificial defect, jumps with $\Delta L=1$ are mostly observed [see Fig. 5(b)]. The reason is that an artificial defect considerably decreases the critical value $p_{c}$ (and hence the field $H_{e n}$-see dotted curve in Fig. 6). From Figs. 2(c) and 3(c) it is clear that the maximum values are $p_{c}^{i d} \simeq 0.54$ for a ring without a defect and $p_{c}^{d} \simeq 0.35$ for a ring with a defect. The ratio $p_{c}^{d} / p_{c}^{i d}$ $\simeq 0.65$ is close to the ratio of the field of first vortex entry $H_{e n}^{d} / H_{e n}^{i d} \simeq 0.67$ obtained from experiment (see Fig. 6). From Fig. 2(c) it is easy to see that for a ring without a defect at $p \simeq 0.35$ there are only jumps with $\Delta L=1$. But if we slightly increase $p$ then jumps with $\Delta L=2$ can appear in the system. So we can conclude that $p=0.35$ is close to the border value which separates regimes with jumps in vorticity of $\Delta L=1$ and $\Delta L=2$. From our experimental data it follows that the maximum value of $p_{c}$ is very close to this border. Thermal fluctuations may influence the value of $\Delta L$, in particular for a $p_{c}$ value close to this border value. This is probably the reason that in the experiment [Fig. 5(b)] occasional jumps with $\Delta L=2$ are observed which are absent in our simulation [Fig. 3(a)].

\section{CONCLUSION}

We studied multiple flux jumps and irreversible behavior of the magnetization of thin mesoscopic Al superconducting rings. We have shown experimentally and theoretically that at low magnetic fields and for rings with sufficiently large radii the vorticity may change by values larger than unity. With increasing magnetic field the order parameter gradually decreases and thus leads to a decrease of the size of the jumps in the vorticity. For rings with width less than $2 \xi$ analytical expressions were obtained for the dependence of the order parameter on the applied magnetic field. We have found that a state with a given vorticity becomes unstable when the value of the gauge-invariant momentum reaches a critical value $p_{c}$ which decreases with increasing magnetic field. This is responsible for the fact that $\Delta L$ decreases with increasing $H$. The introduction of an artificial defect in the ring leads to a decrease of $p_{c}$ in comparison to the case of a ring without a defect and also results in a decrease of $\Delta L$.

\section{ACKNOWLEDGMENTS}

This work was supported by the Flemish Science Foundation (FWO-Vl); the "Onderzoeksraad van de Universiteit Antwerpen;" the "Interuniversity Poles of Attraction Program, Belgian State, Prime Minister's Office, Federal Office for Scientific, Technical, and Cultural Affairs;" EPSRC (UK), and the European ESF Network on Vortex Matter. One of us (D.Y.V.) received individual support from the FWO-Vl. 
*Electronic address: peeters@uia.ua.ac.be

${ }^{\dagger}$ Also at Institute of Microelectronic Technology, Russian Academy of Sciences, Chernogolovka 142432, Russia.

*Electronic address: geim@man.ac.uk

${ }^{1}$ S. Pedersen, G.R. Kofod, J.C. Hollingbery, C.B. Sorensen, and P.E. Lindelof, Phys. Rev. B 64, 104522 (2001).

${ }^{2}$ A.K. Geim, I.V. Grigorieva, S.V. Dubonos, J.G.S. Lok, J.C. Maan, A.E. Filippov, and F.M. Peeters, Nature (London) 390, 259 (1997).

${ }^{3}$ R.J. Zieve, T.F. Rosenbaum, H.M. Jaeger, G.T. Seidler, G.W. Crabtree, and U. Welp, Phys. Rev. B 53, 11849 (1996).

${ }^{4}$ K. Behina, C. Capan, D. Mailly, and B. Etienne, J. Low Temp. Phys. 117, 1435 (1999).

${ }^{5}$ C. Bolech, Gustavo C. Buscaglia, and A. Lopez, Phys. Rev. B 52, R15 719 (1995).

${ }^{6}$ D.Y. Vodolazov, I.L. Maksimov, and E.H. Brandt, Europhys. Lett. 48, 313 (1999).

${ }^{7}$ H.J. Fink and V. Grünfeld, Phys. Rev. B 22, 2289 (1980).

${ }^{8}$ B.J. Baelus, F.M. Peeters, and V.A. Schweigert, Phys. Rev. B 63, 144517 (2001).

${ }^{9}$ J. Berger, Phys. Rev. B 67, 014531 (2003).

${ }^{10}$ D.Y. Vodolazov and F.M. Peeters, Phys. Rev. B 66, 054537
(2002).

${ }^{11}$ D.E. McCumber and B.I. Halperin, Phys. Rev. B 1, 1054 (1970).

${ }^{12}$ M.B. Tarlie and K.R. Elder, Phys. Rev. Lett. 81, 18 (1998).

${ }^{13}$ V.L. Ginzburg, Zh. Éksp. Teor. Fiz. 34, 113 (1958) [Sov. Phys. JETP 7, 78 (1958)].

${ }^{14}$ D.H. Douglass, Jr., Phys. Rev. 124, 735 (1961).

${ }^{15}$ D.Y. Vodolazov and F.M. Peeters, cond-mat/0201564 (unpublished).

${ }^{16}$ A.K. Geim, S.V. Dubonos, J.G.S. Lok, I.V. Grigorieva, J.C. Maan, L.T. Hansen, and P.E. Lindelof, Appl. Phys. Lett. 71, 2379 (1997).

${ }^{17}$ A.K. Geim, S.V. Dubonos, J.J. Palacios, I.V. Grigorieva, M. Henini, and J.J. Schermer, Phys. Rev. Lett. 85, 1528 (2000).

${ }^{18}$ F.M. Peeters and X.Q. Li, Appl. Phys. Lett. 72, 572 (1998).

${ }^{19}$ K.S. Novoselov, A.K. Geim, S.V. Dubonos, Y.G. Cornelissens, F.M. Peeters, and J.C. Maan, Phys. Rev. B 65, 233312 (2002).

${ }^{20}$ P. Singha Deo, V.A. Schweigert, F.M. Peeters, and A.K. Geim, Phys. Rev. Lett. 79, 4653 (1997).

${ }^{21}$ P. Singha Deo, V.A. Schweigert, and F.M. Peeters, Phys. Rev. B 59, 6039 (1999); P. Singha Deo, F.M. Peeters, and V.A. Schweigert, Superlattices Microstruct. 25, 187 (1997). 